\title{
Collaboration in Virtual and Augmented Reality: A Systematic Overview
}

\author{
Catlin Pidel and Philipp Ackermann \\ Zurich University of Applied Sciences \\ Zurich, Switzerland \\ \{catlin.pidel,philipp.ackermann\}@zhaw.ch
}

\begin{abstract}
This paper offers a systematic overview of collaboration in virtual and augmented reality, including an assessment of advantages and challenges unique to collaborating in these mediums. In an attempt to highlight the current landscape of augmented and virtual reality collaboration (AR and VR, respectively), our selected research is biased towards more recent papers (within the last 5 years), but older work has also been included when particularly relevant. Our findings identify a number of potentially under-explored collaboration types, such as asynchronous collaboration and collaboration that combines AR and VR. We finally provide our key takeaways, including overall trends and opportunities for further research.
\end{abstract}

Keywords: augmented reality $\cdot$ virtual reality $\cdot$ collaboration

\section{Introduction}

The idea behind this paper is to better understand the current landscape of collaboration in augmented and virtual reality - to see what is working, what is not, and where further innovations can lead to improvements.

\subsection{Types of Collaboration}

For the purposes of this paper, collaboration can be broken down along two axes synchronous versus asynchronous, and remote versus on-site. A task's synchronicity refers to when collaborators can make contributions (e.g. simultaneously or separately), whereas its locale (remote versus on-site) refers to where the collaborators are.

All possible combinations of these two dichotomies yield four types of collaboration, outlined below.

- Synchronous and On-Site: Collaborators are working together in realtime in the same physical space. Examples include social events, university lectures, and inperson meetings.

- Synchronous and Remote: Collaborators work together in real time, but not from the same physical location. Examples include video and phone conferencing, simultaneous document editing (such as with Google Docs), and live chat. 
- Asynchronous and Remote: This covers many of today's go-to communication tools, such as messaging and email. Collaborators can work when and where is most convenient for them.

- Asynchronous and On-Site: A less common type of collaboration where the location itself is important, but collaborators do not need to be present at the same time. More physically focused projects, such as a construction site with workers on different shifts, fit this definition.

Each of these collaboration types come with tradeoffs. Asynchronous and remote tools, such as email, allow for quick and easy dissemination of information. This very characteristic also makes them prone to informational overload and miscommunication [24], however, especially so when users are juggling several accounts [14]. Alternatively, in-person meetings are less prone to distractions and confusion, but finding a time and place can quickly become an intractable problem. Our goal is to understand these types of collaboration through the lens of virtual and augmented reality, as well as any technology-specific tradeoffs.

\subsection{Paper Structure}

This paper categorizes relevant work into the aforementioned four categories. However, it is important to note that collaboration can transition rather fluidly between these states. Google Docs works as both a synchronous and asynchronous collaboration tool, for example. Similarly, meetings often contain a mixture of co-located and telecommuting participants, thus muddying the waters between "on-site" and "remote". As such, relevant products and research rarely fit neatly into a singular category, so different aspects of papers may be mentioned in multiple sections.

For each section, related work is broken down by whether it applies to VR, AR, or AR/VR-Mixed collaboration. For the purposes of this paper, we define AR to be any computer-generated images overlaid onto the real world, whether a "flat" user interfaces or 3D objects. The latter is sometimes referred to as mixed reality (MR). The VR and AR sections cover head-mounted displays (HMDs), as well as mobile and desktop experiences. These experiences may take place entirely in one technology (VR/VR and AR/AR, respectively), or multiple mediums (e.g. AR and video conferencing). Finally, we have a "AR/VR-Mixed" category for collaboration that combines AR and VR.

\subsection{Contributions}

This paper surveys the current state of collaboration in virtual and augmented realities in both academia and industry.

\section{Synchronous and Remote Collaboration}

\subsection{Synchronous, Remote, VR Collaboration}

Cohabiting a virtual space despite physical distance is a classic use case for virtual reality. Of course, the types of interactions that fit these criteria are extremely varied, and 
the features required for each will vary with the context. Tools like Meetingroom.io [32] are optimized for more professional settings, whereas social collaboration may be more suited to experimental platforms, such as VRChat [56] and AltspaceVR [33]. Virtual reality conferencing affords more social interaction than video conferencing, such as the ability to organically break off into small groups, or interacting with virtual objects in the scene. For example, conducting a design review via VR conferencing allows participants to view and interact with 3D models more intuitively, as well as at real-world scale.

Multiplayer VR games is also a growing industry, with everyone from indie developers to AAA studios releasing titles. For example, "Star Trek: Bridge Crew" [55] and "Space Junkies" [54] are both multiplayer VR games from Ubisoft, an AAA gaming studio. And while many games and social spaces allow for participation via DesktopVR, a growing number require a head-mounted display (HMD), such as the desktop-tethered HTC Vive [22] or standalone Oculus Quest [17]. The market for VR is anticipated to grow even more with the release of $5 \mathrm{G}$ [43], so this is a particular area of opportunity.

In terms of research, one of the main factors in creating VR immersion is the idea of presence - the feeling of truly being in a space. A variety of metrics have been studied for their corresponding effect on presence, including the method of moving around in a virtual world [48] and the relative realism of a user's own avatar when looking in a virtual mirror [57]. Taking this idea a step further, then, a key component of a collaborative virtual experience is thus co-presence.

For example, how does a partner's avatar affect the feeling of connection between two VR collaborators? The feeling of trust? Jo et al. [25] did a number of experiments on how the realism of both an avatar and the surrounding environment affects feelings of co-presence and trust in a teleconference setting. They created cartoonized and photorealistic avatars for participants, and overlaid them onto a backdrop that was similarly cartoonized (3D reconstruction of office) or realistic (actual video feed of office). For clarity, the experimental setup is visually represented in Figure 1 below. Their experiments showed that a realistic (AR) background created more presence, and a cartoon avatar created stronger co-presence than a realistic one. This is consistent with older work on the topic, showing

\begin{tabular}{|c|c|c|}
\hline $\begin{array}{l}\text { Test } \\
\text { ondition }\end{array}$ & Avatar type & $\begin{array}{c}\text { Form of } \\
\text { background }\end{array}$ \\
\hline $\begin{array}{c}\text { 2DV } \\
\text { (Baseline) }\end{array}$ & $\begin{array}{c}\text { Conventional 2D } \\
\text { video based }\end{array}$ & $\begin{array}{c}\text { Conventional } \\
\text { 2D video based }\end{array}$ \\
\hline R-AR & $\begin{array}{l}\text { Realist } \\
\text { censtrots }\end{array}$ & $\begin{array}{c}\text { Real environment } \\
\text { (video backoround) }\end{array}$ \\
\hline C-AR & $\begin{array}{l}\text { Character-like } \\
\text { avatar }\end{array}$ & $\begin{array}{l}\text { Real environment } \\
\text { (video backroround }\end{array}$ \\
\hline R-VR & Reali & \\
\hline & $\begin{array}{l}\text { Characterl-like } \\
\text { avatar }\end{array}$ & environmer \\
\hline
\end{tabular}

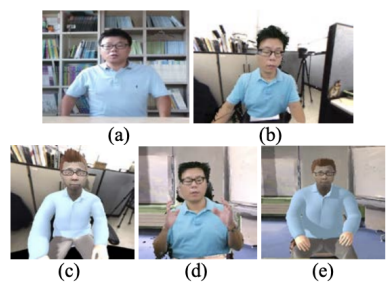

Fig. 1. All iterations of Jo et al.'s work to test how avatar realism affects co-presence and trust [25].

that hyper-realistic avatars can create a

loss of presence through Uncanny Valley [13]. However, when it came to matters of trust, Jo et al. found realistic avatars to be more trustworthy in the eyes of participants. Given these opposing findings, the type of collaborative task (e.g. social versus pro- 
fessional collaboration) should help determine whether co-presence or trust is more important for the situation.

In fact, co-presence may not even be that important for some collaborative use cases. The context of a task - such as whether it's conversation- or task-based, social or professional, one-on-one or in a large group - will affect the requirements of a tool used for that purpose. As noted by Steed and Schroeder [50], realistic avatar gaze may be important for one-on-one conversations in virtual reality, but avatar distinctiveness is probably more important than realism when it comes to collaborating in large groups. Another study by Roth et al. explored how avatar realism affects performance on a verbal task, ultimately showing that the absence of certain social cues (such as eye contact and facial expressions) shifts the user's attention to other cues (such as tone of voice), and does not impede the task's execution [44]. Other findings have corroborated this, as well as showing that people will adjust their own behavior to compensate for technological limitations [50]. This includes compensations such as narrating movements when a user does not think their collaborator can see them, or exaggerating emotional tone if their avatar does not show facial expressions.

\subsection{Synchronous, Remote, AR Collaboration}

Augmented Reality (AR) technology using head-mounted displays (HMD) as well as handheld devices has been used as a tool for supporting collaboration, especially spatial problem solving and remote guidance. It allows for expert guidance from afar, such as for remote surgical consults [15] or remote maintenance [1,39]. One paper, by Kim et al. [27], explored how augmented reality markers can increase the efficiency of remote collaborators solving a tangram puzzle. Using SLAM-based tracking, the remote user was able to contribute spatial cues in addition to their voice, which correlated with significantly higher feelings of connectedness and co-presence for both local and remote participants.

\subsection{Synchronous, Remote, AR/VR-Mixed Collaboration}

A number of studies have found that AR and VR collaboration create better co-presence than traditional video conferencing [25,27], and a number of startups have built products based on this premise. Spatial [49], for example, allows collaboration to join a meeting via AR, VR, or desktop. Their promotional videos promise walls filled with virtual post-it notes and collaborators with photorealistic avatars. Along with these early-adopters, however, there are also critics. Some have argued that augmented or virtual conferencing is most likely unnecessary, even counter-productive, for most use cases, and that the expensiveness of the technology is not worth the purported improvement over traditional methods [16]. That said, Spatial recently announced a \$14 million funding round, indicating not everyone shares this viewpoint [52]. There are also a number of enterprise AR solutions, such as Re'Flekt [41], that use desktop or tablet screens to collaborate with on-site AR workers. One opportunity for these tools would be to instead use VR scene reconstructions for remote collaborators. This could allow for more natural and clear collaboration than 2-dimensional arrows and circles. 
Another interesting project is CoVAR [40], which experiments with realtime collaboration between a local user in AR and a remote user in a VR reconstruction of the local room. This setup allows for interesting applications, such as allowing the VR participant to drastically change their size relative to the room (e.g. "God Mode" and "Mini Mode"), which could have applications in both games and accessibility. Similarly, Zillner et al. [59] developed a SLAM-based method for incredibly accurate scene reconstruction (up to $1 \mathrm{~cm}$ precision) using a specialized headset. This could facilitate remote guidance in VR rather than from a desktop or tablet.

Finally, while not exactly combining AR and VR, Müller et al. [36] conducted a user study comparing performance between AR/AR and VR/VR configurations for distributed (and co-located, discussed in Section 5.3) collaboration using mobile devices. Their qualitative results found advantages and drawbacks of both approaches. The AR setup was helpful in avoiding collisions with real-world objects, while VR conversely was more prone to collisions. Some users also mentioned they were easily disoriented when they looked away from the screen during the VR experience (which was necessary to avoid tripping) because there was no visual pairing between the VR and actual worlds. On the other hand, AR struggled where VR excelled - AR collaborators could not reference a virtual object's location relative to their own physical space (despite being visually integrated with the task), but VR users benefitted from seeing the same (virtual) world. Despite these tradeoffs, there was no statistically significant difference between performance or users' mental workload for either condition, and user preference was evenly split between AR and VR. Based on these findings, the authors recommend transitional interfaces that go between AR and VR. This could be a compelling way for a single tool to leverage the relative benefits of AR and VR for different contexts.

\section{Asynchronous and Remote Collaboration}

\subsection{Asynchronous, Remote, VR Collaboration}

Virtual reality training sessions allow for expert knowledge to be transferred asynchronously and remotely. This has been used quite frequently in both military and medical sectors. Bhagat et al. found that using virtual reality for weapons firing training was more cost-effective, improved learning outcomes, and allowed for more individualized training (since the VR experience could keep track of students' metrics over time) [7]. There have also been multiple studies showing that VR simulations improve operating room performance across a variety of surgery types [45,53].That said, training sessions are only loosely collaborative in that the flow of information is, most often, one-way one curated experience can disseminate information to a vast number of students, but the student has little to no affect on it.

For asynchronous collaboration between two or more virtual reality users, however, the pool of research is noticeably smaller. A 2013 chapter of Mental Health Informatics discussed the potential of asynchronous psychotherapy, which is currently gaining popularity with text-based therapy apps like BetterHelp [6], as well as VR applications to aid in treatment for a variety of illnesses, such as VR exposure therapy (VRE) for those with post-traumatic stress disorder (PTSD) or a particular phobia [30]. While not 
mentioned in this paper, a combination of VR-based, asynchronous therapy could be an interesting direction.

Another interesting application is the idea of an asynchronous, virtual classroom, as proposed by Morozov et al. [35]. Their system created a virtual classroom where each student could virtually attend a pre-recorded class, either by observing or re-recording the class with their own input (e.g. to class discussions). This allows for asynchronous class discussions that, though limited, could be an interesting way to encourage collaboration between in-person coursework and remote learners.

\subsection{Asynchronous, Remote, AR Collaboration}

Similar to virtual reality, there is little work on asynchronous, remote collaboration between multiple AR users. Given that AR is largely location-based, it makes sense that there would be limited use cases for remote (aka location-independent) augmented reality. One interesting example is the Blocks app, which explores co-creation between augmented and virtual reality in a variety of collaboration types, including asynchronous and remote scenarios [20]. While it does fall under the "asynchronous, remote AR" label, it also fits any of the other categories mentioned in this paper - the app allows users to collaboratively build sculptures with virtual blocks, and it's built to work synchronously, asynchronously, on-site, and remote in either AR or VR.

\subsection{Asynchronous, Remote, AR/VR-Mixed Collaboration}

While there are, again, few papers in this realm, there are use cases that could fit this sort of collaboration. For example, interior design generally requires at-home visits, but using augmented-reality room scanning, designers could design a room in VR that clients could review in their home using AR. This could allow for easier visualization for clients, and to give feedback on designs without needing a scheduled meeting.

\section{Asynchronous and On-Site Collaboration}

\subsection{Asynchronous, On-Site, VR Collaboration}

We have been unable to find products or papers that present a use case for this sort of VR collaboration. While some virtual reality experiences are location-dependent, such as in the case of Cave Automatic Virtual Environments (CAVE) experiences, we have found very little current research (since the year 2000) on collaboration in these types of spaces, and virtually nothing asynchronous in nature. While interesting research and use cases could exist outside our knowledge, we still do not find this area to be of very high priority for further research and development.

Applications that combine VR and AR are similarly sparse for this form of collaboration, so that subsection has been excluded from this section. 


\subsection{Asynchronous, On-Site, AR Collaboration}

Augmented reality allows users to place labels to communicate important locations and/or information. This is particularly useful when collaborators need to relay information about a location (e.g. factory floor, shared apartment, construction site), but cannot do so in person. For example, Renevier and Nigay's MAGIC (Mobile Augmented Group Interaction in Context) project shows how AR can be used to label sites in an archaeological dig with important information, thus allowing for teams to work asynchronously [42]. Similarly, multiple papers have discussed various augmentedreality museum guides, allowing for visitors to interact and learn about exhibits at their own pace $[18,21]$. This is a particularly exciting area, since products like Microsoft's HoloLens [34] and Google's industry-rebranding of Glass [19] are making this sort of technology more accessible to the public.

AR collaboration can also move beyond simply placing markers, however. Irlitti et al. make the distinction between direct and passive interactions, arguing that the latter holds a lot of opportunity, despite the current lack of academic exploration [23]. In their paper, direct interactions are defined as information the user explicitly creates (e.g. markers), whereas passive interactions are information that can be collected by the application itself, such as the time spent at certain locations, or how a user moves through a space.

\section{Synchronous and On-Site Collaboration}

\subsection{Synchronous, On-Site, VR Collaboration}

Collocated VR comes with some challenges, such as avoiding collisions between users, and really only makes sense when collaborators need to directly interact with each other in a virtual world. That said, it also has some advantages over remote VR, such as the potential for offline and latency-free collaboration. It also allows the experience to rely on virtually-tracked props, which has been shown to aid in suspension of disbelief (provided the physical props are similar in texture and weight to their virtual representation), a practice sometimes called "substitutional reality" [47]. Seen in Figure 2 below, this sort of physical/virtual mapping is already being seen on a commercial level with brickand-mortar virtual reality centers, offering group activities such as VR adventure games and escape rooms designed specifically for the space.

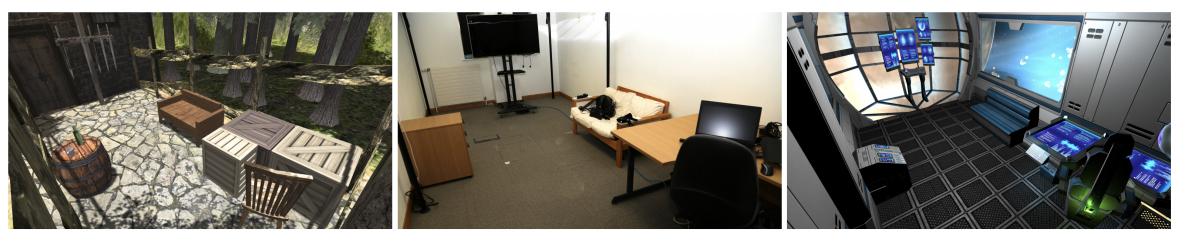

Fig. 2. Example of a physical space mapped to a virtual world, from Simeone et al.'s work on Substitutional Reality [47]. 
An important research area of co-located VR is avoiding collisions when two or more users are occupying the tracking area. Especially if the virtual world is larger than the physical space, two users can physically collide despite being in different areas of the virtual world. Lacoche et al. experimented with different methods of displaying someone's physical location in VR, including two types of avatars and a "Safe Navigation Floor" (SNF) that highlighted the ground in green or red depending on whether the physical location was occupied or not. Their results showed fewer collisions with avatars relative to the SNF approach, and user sentiment also preferred this setup to the potentially safer approach of separating users into two individual, but smaller, tracking areas [28]. A similar study by Azmandian et al. experimented with redirected walking to prevent user collisions with positive results, though the degree of success was heavily influenced by the size and shape of the physical space [5].

Finally, there are some relevant collaborations that mix virtual reality and actual reality. The video game "Keep Talking and Nobody Explodes", for example, is a cooperative game where a player in VR needs to communicate information to their non-VR counterpart [51]. Another is a paper by Mai et al., which allows for communication between HMD- and non-HMD-wearing users through a shared surface. Cameras above a table allow for the VR participant to see images from the real world without having to remove their HMD, including video overlay of the other participants hands. In a user study using this shared surface, the non-VR participant needed to explain a series of steps to the VR participant, and the presence of hands halved the number of clarifying questions needed to complete the task [31]. While these are not true VR-to-VR collaborations, they are good examples of highly-collaborative interactions that rely heavily on VR.

\subsection{Synchronous, On-Site, AR Collaboration}

Early research, such as Billinghurst's Shared Space experiment [11], showed the potential for augmented reality to enhance face-to-face communication by combining the task space (such as a computer screen or 3D model) with the collaboration space (faceto-face communication) $[9,10]$. Today, multi-user AR has become much more common, with popular apps like Pokemon Go [37], and AR development kits from both Google and Apple that simplify the creation of AR apps. Apple's ARKit, for example, makes it quite easy for iOS developers to get realtime, multi-user AR up and running [2]. This technology was featured in the form of a competitive AR slingshot game during the WWDC18 keynote [3].

Augmented reality collaboration has also been used for art exhibitions. Artechouse, a Washington-DC-based experimental art studio, frequently incorporates both phoneand projector-based augmented reality into their exhibits [4].

A particularly interesting example of synchronous AR collaboration is CARS (Collaborative Augmented Reality for Socialization) developed by Zhang et al. [58]. It allows for information to be shared between nearby phones, allowing for lower latency as well as leveraging the computing power of multiple devices to create higher quality AR experiences. While this is a framework rather than an standalone product, it could improve the experience of future AR mobile applications. 


\subsection{Synchronous, On-Site, AR/VR-Mixed Collaboration}

This is another section where research is, perhaps intentionally, sparse. Collaboration between augmented and virtual reality users occupying the same physical space would require a very specific use case. One such use case is the work by Billinghurst et al. to create a "Magic Book" [8]. The book, which looks like a normal children's book, uses image recognition and an HMD to superimpose virtual objects that "pop off" the page. These scenes can then be "entered" and the glasses transition from AR to VR mode for a first-person view of the scene. Multiple readers can enjoy a story together, creating a very interesting collaborative experience - users in VR mode are represented as avatars in the scene and visible by fellow VR collaborators, and also from a "bird's eye view" to those still in AR mode. Other research has explored transitioning between VR and a workstation environment [26], which could aid in collaboration, but the paper was not explicitly collaborative in focus. While fascinating, it's also worth noting that these prior examples were all published at least 10 years ago, so there's room for more research with more current technologies. We have been unable to find research on collaboration in mixed realities, other than the Blocks app mentioned in Section 3.2.

That said, there has been research comparing AR/AR and VR/VR configurations for co-located (and distributed, discussed in Section 2.3) collaborative tasks [36]. In this paper, Müller et al. conduct an analysis of a turn-based matching game, comparing quantitative performance and qualitative user preferences between the configurations. VR showed a slight, but significant decrease in the number of trials to solve the task, but there was otherwise no significant performance difference between the AR and VR scenarios. Qualitatively, participants' preference was fairly evenly split between AR and VR, but their opinions were strongly held. Those who preferred AR said it was helpful to see their collaborator on the screen, and thus easier to coordinate. Fans of VR found AR distracting, and thought the "tidiness" of VR made it more immersive and easier to focus. One way to address these strong but opposing preferences would be to allow users to switch fluidly AR and VR - a term Müller et al. refer to as transitional reality.

\section{Additional Research of Note}

This section covers related work that does not otherwise fit into one of our predefined categories, but is still relevant to AR/VR collaboration.

Being able to direct a user's attention is incredibly important for collaboration, regardless of the type. Synchronous collaboration requires getting collaborators attention, and asynchronous collaboration needs to represent changes since the last time a user was in a virtual environment. In the realm of AR, Biocca et al. developed an AR "attention funnel" that uses a chain of successively smaller rectangles to direct a user's gaze to a specified point, even if it's outside their current field of view [12]. VR also had a number of papers, especially related to directing attention in $360^{\circ}$ video. One study by the $\mathrm{BBC}$ showed that a combination of audio and visual cues (in this case, a sound coming from the out-of-frame location, followed by a person in-frame walking towards the sound) was most successful at guiding a user's attention [46]. Another compared a similar situation (a firefly flying toward the target location) with forcibly rotating the 
video to display the relevant area, but neither showed any significant difference from the control condition (no guidance) [38].

Another interesting question is how to define "remote collaboration". In our research, we define it as two or more parties in separate physical locations. However, distance between collaborators is more than just geographical distance, as illustrated by the Virtual Distance metric developed by Dr. Karen Sobel Lojeski [29]. In addition to physical distance, it also encompasses a wide variety of factors, such as cultural and emotional distance, that can impede productivity between collaborators. While this paper uses the more binary definition of "remote work", it is important to note that not all distance can be bridged by faster computers or better immersive technology.

\section{Discussion}

Based on this literature review, we have identified four areas with little or no current research, as shown in Table 1.

\begin{tabular}{|c|c|}
\hline Synchronous & Asynchronous \\
\hline $\begin{array}{l}\text { VR: } \\
\text { Social and gaming experiences }[32,33,54,55 \text {, } \\
56], 5 \mathrm{G} \text { facilitating VR }[43], \text { presence/co-presence } \\
{[13,25,44,48,50,57], \text { compensating for missing }} \\
\text { social cues, Blocks app [20]. }\end{array}$ & $\begin{array}{l}\text { VR: } \\
\text { VR training (military, medical) }[7,45,53], \text { VR } \\
\text { exposure therapy and other therapeutic } \\
\text { applications [6,30],"vacademia" virtual classroom } \\
\text { [35], Blocks app [20]. }\end{array}$ \\
\hline $\begin{array}{l}\text { AR: } \\
\text { Tangram-puzzle collaboration }[27], \text { remote } \\
\text { guidance }[1,15,39] \text {, Blocks app }[20]\end{array}$ & $\begin{array}{l}\text { AR: } \\
\text { Limited existing work (other than Blocks app [20]). }\end{array}$ \\
\hline $\begin{array}{l}\text { AR/VR-Mixed: } \\
\text { VR/AR have better co-presence than video [25, } \\
27], \text { MR conferencing }[16,41,49,52] \text {, CoVAR }[40] \text {, } \\
\text { hyper-accurate scene reconstruction for remote } \\
\text { guidance [59], Blocks app [20]. }\end{array}$ & $\begin{array}{l}\text { AR/VR-Mixed: } \\
\text { Limited existing work (other than Blocks app [20]). }\end{array}$ \\
\hline $\begin{array}{l}\text { VR: } \\
\text { Substitutional reality [47], avoiding collisions [5, } \\
\text { 28], "Keep Talking and Nobody Explodes" [51], } \\
\text { Shared Surfaces [31], Blocks app [20]. }\end{array}$ & $\begin{array}{l}\text { VR: } \\
\text { Limited existing work (other than Blocks app [20]). }\end{array}$ \\
\hline $\begin{array}{l}\text { AR: } \\
\text { Shared Space [11], task vs. collaboration space } \\
{[9,10], \text { games }[3,37], \text { ARKit }[2], \text { art exhibitions }} \\
{[4], \text { CARS framework [58], Blocks app [20]. }}\end{array}$ & $\begin{array}{l}\text { AR: } \\
\text { MAGIC (tool for annotating archeological dig sites) } \\
{[42], \text { AR museum guides }[18,21] \text {, collaboration by }} \\
\text { tracking passive information (e.g. how someone } \\
\text { moves through a space) [23]. }\end{array}$ \\
\hline $\begin{array}{l}\text { AR/VR-Mixed: } \\
\text { Magic Book [8], work-space/VR transitional } \\
\text { headset [26], Blocks app [20], transitional reality } \\
{[26] .}\end{array}$ & $\begin{array}{l}\text { AR/VR-Mixed: } \\
\text { Limited existing work (other than Blocks app [20]). }\end{array}$ \\
\hline
\end{tabular}

Table 1. Summary of topics discussed in this paper. There are four areas that are currently under explored. 
We have also reflected on the unique tradeoffs to collaborating in augmented and virtual reality, and will outline a number of advantages and disadvantages based on our research and prior experience in augmented and virtual reality.

\section{Benefits of Collaboration in Virtual and Augmented Reality}

- Being able to collaborate independently in both time and space gives freedom, but also increases the user's mental load. Similar to multiple versions of a co-edited document, there can be "save points" in AR/VR time, which have potential to be both useful and confusing. This area is currently under explored, and could benefit from further research.

- Visualizations and other immersive experiences make it easier to communicate a concept, meaning less reliance on people's imagination. This is especially helpful in areas like interior design and architecture.

- A truly engaging, immersive AR/VR experience could dramatically improve the quality of remote collaboration, and companies like Spatial are already working towards that end. However, avatar quality is a limiting factor to co-presence. They are often too cartoonish for business purposes, or photorealstic to the point of verging on Uncanny Valley. Commercially available avatars that realistically mimic users' expressions (e.g. gaze, mouth movement) would be a game-changer, though this is reliant on both software and hardware improvements.

- Virtual and augmented reality allow for viewing things from a different user's perspective. For example, being able to see a room through a color blindness filter, or adjusting a user's height to view a space from a child's height. This could be used advantageously for empathy training, as well as a new method of accessibility testing in architecture and design.

\section{Challenges Facing Collaboration in Virtual and Augmented Reality}

- Expensive and rapidly evolving technology limits who can participate. This is a problem both from an equality as well as a market size standpoint. Mobile augmented reality with smartphones and consumer-oriented virtual reality headsets are still the most easily accessible.

- Motion sickness and eye fatigue are ongoing problems, further limiting the potential audience for VR and AR applications.

- In teleconferencing settings, the additional complexity of virtual or augmented reality meetings translates to more points for system or user error. There is only so much technical literacy that can be assumed of the average consumer. This will of course increase with time and exposure, but it could lead to lower adoption due to frustration.

- Rapidly changing technology as well as a large variety of platforms (Oculus, Vive, Cardboard, WebVR, mobile, etc) can substantially increase development overhead for a cross-platform application, and focusing on only one platform significantly limits the potential audience.

- A single collaborative task may require several mediums, such as switching between face-to-face discussion, viewing slides or a 3D model, and taking notes. Especially in virtual reality, these sorts of context switches often require taking a bulky headset on and off, which can interrupt the flow of work. 
- There's currently no industry standard for drawing someone's attention to something outside their field of vision. This is especially important for asynchronous collaboration - how can we alert the user that something has changed since the last time they were in a virtual/augmented space?

\subsection{Conclusions}

Virtual and augmented reality collaboration have made some compelling advancements in recent years, but there's still plenty of room for improvement and further exploration. Asynchronous collaboration, for example - whether in augmented or virtual, remote or on-site settings - is particularly under explored. This paper identifies four areas of opportunity, as outlined in Table 1, all of which fall under the asynchronous category. Experiences that combine virtual and augmented reality are also relatively rare, and could benefit from further work.

Based on these findings, the authors propose a number of potential topics for further study. In the vein of asynchronous collaboration, there is relatively little work into how to represent changes to a virtual world - similar to how changes are highlighted in Github, how can changes by one collaborator be represented to another, asynchronous collaborator? While implementation may vary by use case, this seems to be a limiting factor to asynchronous collaborative experiences. For AR/VR-Mixed research, opportunities include applications with both AR and VR participants, as well as transitional interfaces that would allows a single participant to go between virtual and augmented experiences. Ultimately, augmented and virtual collaboration hold a lot of potential, which we hope to explore further in future work.

\section{References}

1. Adcock, M., Gunn, C.: Annotating with'sticky'light for remote guidance. In: ACM SIGGRAPH ASIA 2010 Posters, pp. 1-1 (2010)

2. Apple Inc.: Creating a multiuser ar experience (2020), https://developer.apple.com/documentation/arkit/ creating_a_multiuser_ar_experience

3. Apple Inc.: Swiftshot: Creating a game for augmented reality (2020), https://developer.apple.com/documentation/arkit/

swiftshot_creating_a_game_for_augmented_reality

4. Artechouse: Artechouse (2019), https: / / www.artechouse.com/

5. Azmandian, M., Grechkin, T., Rosenberg, E.S.: An evaluation of strategies for two-user redirected walking in shared physical spaces. In: 2017 IEEE Virtual Reality (VR). pp. 91-98. IEEE (2017)

6. BetterHelp: Betterhelp (2020), https : / /www.betterhelp.com/

7. Bhagat, K.K., Liou, W.K., Chang, C.Y.: A cost-effective interactive $3 \mathrm{~d}$ virtual reality system applied to military live firing training. Virtual Reality 20(2), 127-140 (2016)

8. Billinghurst, M., Cambell, S., Poupyrev, I., Kato, K.T.H., Chinthammit, W., Hendrickson, D.: Magic book: Exploring transitions incollaborative arinterfaces. In: Proc. of SIGGRAPH. p. 87 (2000)

9. Billinghurst, M., Belcher, D., Gupta, A., Kiyokawa, K.: Communication behaviors in colocated collaborative ar interfaces. International Journal of Human-Computer Interaction 16(3), 395-423 (2003) 
10. Billinghurst, M., Kato, H.: Collaborative augmented reality. Communications of the ACM 45(7), 64-70 (2002)

11. Billinghurst, M., Weghorst, S., Furness, T.: Shared space: An augmented reality approach for computer supported collaborative work. Virtual Reality 3(1), 25-36 (1998)

12. Biocca, F., Tang, A., Owen, C., Xiao, F.: Attention funnel: omnidirectional 3d cursor for mobile augmented reality platforms. In: Proceedings of the SIGCHI conference on Human Factors in computing systems. pp. 1115-1122 (2006)

13. Brenton, H., Gillies, M., Ballin, D., Chatting, D.: The uncanny valley: does it exist and is it related to presence. Presence connect (2005)

14. Cecchinato, M.E., Sellen, A., Shokouhi, M., Smyth, G.: Finding email in a multi-account, multi-device world. In: Proceedings of the 2016 CHI Conference on Human Factors in Computing Systems. pp. 1200-1210 (2016)

15. Davis, M.C., Can, D.D., Pindrik, J., Rocque, B.G., Johnston, J.M.: Virtual interactive presence in global surgical education: international collaboration through augmented reality. World neurosurgery 86, 103-111 (2016)

16. Dice: Augmented reality collaboration is real. avoid it like the plague (2019), https:// insights.dice.com/2019/01/25/augmented-reality-collaboration/

17. Facebook Technologies, LLC.: Oculus quest (2020), https://www.oculus.com/ quest $/$ ? locale=en_Us

18. Fenu, C., Pittarello, F.: Svevo tour: The design and the experimentation of an augmented reality application for engaging visitors of a literary museum. International Journal of HumanComputer Studies 114, 20-35 (2018)

19. Google: Glass (2019), https: / / www.google.com/glass/

20. Guo, A., Canberk, I., Murphy, H., Monroy-Hernández, A., Vaish, R.: Blocks: Collaborative and persistent augmented reality experiences. Proceedings of the ACM on Interactive, Mobile, Wearable and Ubiquitous Technologies 3(3), 1-24 (2019)

21. Hammady, R., Ma, M., Temple, N.: Augmented reality and gamification in heritage museums. In: Joint International Conference on Serious Games. pp. 181-187. Springer (2016)

22. HTC Corporation: Htc vive (2020), https : / / www.vive.com/eu/

23. Irlitti, A., Smith, R.T., Von Itzstein, S., Billinghurst, M., Thomas, B.H.: Challenges for asynchronous collaboration in augmented reality. In: 2016 IEEE International Symposium on Mixed and Augmented Reality (ISMAR-Adjunct). pp. 31-35. IEEE (2016)

24. Jackson, T.W., van den Hooff, B.: Understanding the factors that effect information overload and miscommunication within the workplace. Journal of Emerging Trends in Computing and Information Sciences 3(8), 1240-1252 (2012)

25. Jo, D., Kim, K.H., Kim, G.J.: Effects of avatar and background types on users' co-presence and trust for mixed reality-based teleconference systems. In: In Proceedings the 30th Conference on Computer Animation and Social Agents. pp. 27-36 (2017)

26. Kijima, R., Ojika, T.: Transition between virtual environment and workstation environment with projective head mounted display. In: Proceedings of IEEE 1997 Annual International Symposium on Virtual Reality. pp. 130-137. IEEE (1997)

27. Kim, S., Lee, G., Sakata, N., Billinghurst, M.: Improving co-presence with augmented visual communication cues for sharing experience through video conference. In: 2014 IEEE International Symposium on Mixed and Augmented Reality (ISMAR). pp. 83-92. IEEE (2014)

28. Lacoche, J., Pallamin, N., Boggini, T., Royan, J.: Collaborators awareness for user cohabitation in co-located collaborative virtual environments. In: Proceedings of the 23rd ACM Symposium on Virtual Reality Software and Technology. pp. 1-9 (2017)

29. Lojeski, K.S.: Virtual Distance ${ }^{\mathrm{TM}}$ : A proposed model for the study of virtual work. Stevens Institute of Technology (2006) 
30. Maghazil, A., Yellowlees, P.: Novel approaches to clinical care in mental health: from asynchronous telepsychiatry to virtual reality. In: Mental Health Informatics, pp. 57-78. Springer (2014)

31. Mai, C., Bartsch, S.A., Rieger, L.: Evaluating shared surfaces for co-located mixed-presence collaboration. In: Proceedings of the 17th International Conference on Mobile and Ubiquitous Multimedia. pp. 1-5. ACM (2018)

32. meethingRoom.io: Virtual meeting rooms (2019), https://meetingroom.io/thevirtual-meeting-rooms-product/

33. Microsoft: Altspacevr (2020), https: / / altvr.com/

34. Micrsoft: Hololens 2: A new vision for computing (2020), https:// www.microsoft.com/en-us/hololens/

35. Morozov, M., Gerasimov, A., Fominykh, M., Smorkalov, A.: Asynchronous immersive classes in a 3d virtual world: Extended description of vacademia. In: Transactions on computational science XVIII, pp. 81-100. Springer (2013)

36. Müller, J., Zagermann, J., Wieland, J., Pfeil, U., Reiterer, H.: A qualitative comparison between augmented and virtual reality collaboration with handheld devices. In: Proceedings of Mensch und Computer 2019, pp. 399-410 (2019)

37. Niantic Inc.: Pokemon go (2020), https://pokemongolive.com/en/

38. Nielsen, L.T., Møller, M.B., Hartmeyer, S.D., Ljung, T.C., Nilsson, N.C., Nordahl, R., Serafin, S.: Missing the point: an exploration of how to guide users' attention during cinematic virtual reality. In: Proceedings of the 22nd ACM Conference on Virtual Reality Software and Technology. pp. 229-232 (2016)

39. Palmarini, R., Erkoyuncu, J.A., Roy, R., Torabmostaedi, H.: A systematic review of augmented reality applications in maintenance. Robotics and Computer-Integrated Manufacturing 49, 215-228 (2018)

40. Piumsomboon, T., Lee, Y., Lee, G., Billinghurst, M.: Covar: a collaborative virtual and augmented reality system for remote collaboration. In: SIGGRAPH Asia 2017 Emerging Technologies, pp. 1-2 (2017)

41. Re'flekt GmbH: Re'flekt (2019), https: / / www.reflekt.com/de/

42. Renevier, P., Nigay, L.: Mobile collaborative augmented reality: the augmented stroll. In: IFIP International Conference on Engineering for Human-Computer Interaction. pp. 299316. Springer (2001)

43. Rogers, S.: 2019: The year virtual reality gets real (2019), https : / www.forbes.com/ sites/solrogers/2019/06/21/2019-the-year-virtual-realitygets-real/

44. Roth, D., Lugrin, J.L., Galakhov, D., Hofmann, A., Bente, G., Latoschik, M.E., Fuhrmann, A.: Avatar realism and social interaction quality in virtual reality. In: 2016 IEEE Virtual Reality (VR). pp. 277-278. IEEE (2016)

45. Seymour, N.E., Gallagher, A.G., Roman, S.A., O'brien, M.K., Bansal, V.K., Andersen, D.K., Satava, R.M.: Virtual reality training improves operating room performance: results of a randomized, double-blinded study. Annals of surgery 236(4), 458 (2002)

46. Sheikh, A., Brown, A., Watson, Z., Evans, M.: Directing attention in 360-degree video (2016)

47. Simeone, A.L., Velloso, E., Gellersen, H.: Substitutional reality: Using the physical environment to design virtual reality experiences. In: Proceedings of the 33rd Annual ACM Conference on Human Factors in Computing Systems. pp. 3307-3316 (2015)

48. Slater, M., Usoh, M., Steed, A.: Taking steps: the influence of a walking technique on presence in virtual reality. ACM Transactions on Computer-Human Interaction (TOCHI) 2(3), 201-219 (1995)

49. Spatial Systems, Inc.: Spatial: Collaborate anywhere in ar (2020), https:// spatial.io/ 
50. Steed, A., Schroeder, R.: Collaboration in immersive and non-immersive virtual environments. In: Immersed in Media, pp. 263-282. Springer (2015)

51. Steel Crate Games, Inc.: Keep talking and nobody explodes (2018), https:// keeptalkinggame.com/

52. TechCrunch: Spatial raises 14 million more for a holographic $3 \mathrm{~d}$ workspace app, a vr/ar version of zoom or hangouts (2020), https://techcrunch.com/2020/01/ $30 /$ spatial-raises-14m-more-for-a-holographic-3d-workspaceapp-a-vr-ar-version-of-zoom-or-hangouts /

53. Thomsen, A.S.S., Bach-Holm, D., Kjærbo, H., Højgaard-Olsen, K., Subhi, Y., Saleh, G.M., Park, Y.S., la Cour, M., Konge, L.: Operating room performance improves after proficiencybased virtual reality cataract surgery training. Ophthalmology 124(4), 524-531 (2017)

54. Ubisoft Entertainment: Space junkies (2017), https://www.ubisoft.com/en-us/ game/space-junkies/

55. Ubisoft Entertainment: Star trek: Bridge crew puts you and your friends in the heart of a starship (2017), https://www.ubisoft.com/en-us/game/star-trek-bridgecrew/

56. VRChat Inc.: Vrchat (2020), https: / /www.vrchat.com/

57. Waltemate, T., Gall, D., Roth, D., Botsch, M., Latoschik, M.E.: The impact of avatar personalization and immersion on virtual body ownership, presence, and emotional response. IEEE transactions on visualization and computer graphics 24(4), 1643-1652 (2018)

58. Zhang, W., Han, B., Hui, P., Gopalakrishnan, V., Zavesky, E., Qian, F.: Cars: Collaborative augmented reality for socialization. In: Proceedings of the 19th International Workshop on Mobile Computing Systems \& Applications. pp. 25-30 (2018)

59. Zillner, J., Mendez, E., Wagner, D.: Augmented reality remote collaboration with dense reconstruction. In: 2018 IEEE International Symposium on Mixed and Augmented Reality Adjunct (ISMAR-Adjunct). pp. 38-39. IEEE (2018) 